\title{
ON A FUNCTIONAL CENTRAL LIMIT THEOREM FOR THE LINEAR PROCESS GENERATED BY ASSOCIATED RANDOM VARIABLES IN A HILBERT SPACE
}

\author{
Mi-Hwa Ko And TAE-Sung Kim
}

\begin{abstract}
Let $\left\{\xi_{k}, k \in \mathbb{Z}\right\}$ be a strictly stationary associated sequence of $H$-valued random variables with $E \xi_{k}=0$ and $E\left\|\xi_{k}\right\|^{2}<\infty$ and $\left\{a_{k}, k \in\right.$ $\mathbb{Z}\}$ a sequence of linear operators such that $\sum_{j=-\infty}^{\infty}\left\|a_{j}\right\|_{L(H)}<\infty$. For a linear process $X_{k}=\sum_{j=-\infty}^{\infty} a_{j} \xi_{k-j}$ we derive that $\left\{X_{k}\right\}$ fulfills the functional central limit theorem.
\end{abstract}

\section{Introduction}

Let $H$ be a separable real Hilbert space with the norm $\|\cdot\|_{H}$ generated by an inner product, $\langle\cdot, \cdot\rangle_{H}$ and let $\left\{e_{k}, k \geq 1\right\}$ be an orthonormal basis in $H$. Let $L(H)$ be the class of bounded linear operators from $H$ to $H$ and denote by $\|\cdot\|_{L(H)}$ its usual norm. Let $\left\{\xi_{k}, k \in \mathbb{Z}\right\}$ be a strictly stationary sequence of $H$-valued random variables, and $\left\{a_{k}, k \in \mathbb{Z}\right\}$ be a sequence of operators, $a_{k} \in L(H)$. We define the stationary Hilbert space process by:

$$
X_{k}=\sum_{j=-\infty}^{\infty} a_{j} \xi_{k-j}, k \in \mathbb{Z} .
$$

The sequence $\left\{X_{k}, k \in \mathbb{Z}\right\}$ is a natural extension of the multivariate linear processes (Brockwell and Davis [5], Chap. 11). These types of processes with values in functional spaces also facilitate the study of estimation and forecasting problems for several classes of continuous time processes. For more details see Bosq [3].

We define

$$
W_{n}(t)=n^{-\frac{1}{2}} \sum_{k=1}^{[n t]} X_{k}, t \in[0,1]
$$

Received August 6, 2007.

2000 Mathematics Subject Classification. 60F05, 60F17, 60G10.

Key words and phrases. functional central limit theorem, linear process in a Hilbert space, association, linear operator, Hilbert space-valued random variable.

This work was partially supported by the Korean Research Foundation Grant funded by Korean Government(KRF-2006-521-C00026, KRF-2006-353-C00006). 
When $\left\{\xi_{k}, k \in \mathbb{Z}\right\}$ is a sequence of $H$-valued i.i.d. random variables such that $E\left\|\xi_{k}\right\|^{2}<\infty$ and $E \xi_{k}=0$ if $\sum_{j=-\infty}^{\infty}\left\|a_{j}\right\|_{L(H)}<\infty$, then the series in (1.1) converges almost surely and in $L_{1}(H)$ (Denisevskii and Dorogovtsev [8]). Moreover, $X_{k}$ satisfies a functional central limit theorem (Bosq [3]) and the Berry-Esseen inequality (Bosq [4]).

A sequence $\left\{\xi_{i}, 1 \leq i \leq n\right\}$ of real-valued random variables is said to be associated if for any coordinatewise increasing functions $f, g: \mathbb{R}^{n} \rightarrow \mathbb{R}$

$$
\operatorname{Cov}\left(f\left(\xi_{1}, \ldots, \xi_{n}\right), g\left(\xi_{1}, \ldots, \xi_{n}\right)\right) \geq 0
$$

whenever this exists. Associated sequences are widely encountered in applications; e.g. in reliability, in mathematical physics and percolation theory (c.f. Barlow and Proschan [1], Newman [11], Cox and Grimmett [7]). Newman [11] proved the central limit theorem, Newman and Wright [12] extended this to a functional central limit theorem.

Recently Kim and Ko [10] derived a functional central limit theorem for the linear process generated by associated random variables as follows.

Theorem 1.1 (Kim and Ko [10]). Let $\left\{\xi_{k}\right\}$ be a strictly stationary sequence of centered and associated random variables having finite second moment and let $\left\{a_{k}\right\}$ be a sequence of numbers such that

$$
\sum_{j=-\infty}^{\infty}\left|a_{j}\right|<\infty .
$$

Define $X_{k}$ by (1.1), $W_{n}$ by (1.2) and assume

$$
\sigma^{2}=E \xi_{1}^{2}+2 \sum_{j=2}^{\infty} E\left(\xi_{1} \xi_{j}\right)<\infty .
$$

Then, as $n \rightarrow \infty$

$$
W_{n}(t) \Rightarrow W^{1},
$$

where $\Rightarrow$ indicates weak convergence and $W^{1}$ is a Wiener process with variance $\left(\sum_{j=-\infty}^{\infty} a_{j}\right)^{2} \sigma^{2}$.

In the studying the infinite-dimensional case, our question is to what extent Theorem 1.1 remains valid in the new context when we replace $\left\{\xi_{k}\right\}$ by an infinite-dimensional space valued random variables, the constants by linear bounded operators and absolute values by the corresponding norms. To see new possible quality effects, we consider a simplest case of infinite dimensional Hilbert space $H$ in this paper.

\section{Preliminaries}

Theorem 2.1 (Newman, Wright [12]). Let $\left\{\xi_{1}, \ldots, \xi_{m}\right\}$ be a sequence of associated random variables with $E\left|\xi_{i}\right|^{2}<\infty$ and $E \xi_{i}=0 i \geq 1$, and let $M_{m}=\max \left(S_{1}, \ldots, S_{m}\right)$, where $S_{m}=\xi_{1}+\cdots+\xi_{m}$. Then

$$
E\left(M_{m}^{2}\right) \leq E\left(S_{m}\right) \text {. }
$$


As the notion of weakly associated random vectors in Burton et al. [6], we introduce the concept of associated random vectors.

Definition 2.2. A finite sequence $\left\{\xi_{i}, 1 \leq i \leq n\right\}$ of $\mathbb{R}^{d}$-valued random vectors is said to be associated if for all coordinatewise increasing functions $f, g: \mathbb{R}^{n d} \rightarrow \mathbb{R}, \operatorname{Cov}\left(f\left(\xi_{1}, \ldots, \xi_{n}\right), g\left(\xi_{1}, \ldots, \xi_{n}\right)\right) \geq 0$ whenever this is defined. An infinite family of $\mathbb{R}^{d}$-valued random vectors is associated if every finite subfamily is associated.

From the functional central limit theorem of weakly associated random vectors in Burton et al. [6], we can obtain the following functional central limit theorem for stationary associated random vectors.

Theorem 2.3. Let $\left\{\xi_{i}, i \geq 1\right\}$ be a strictly stationary associated sequence of $\mathbb{R}^{d}$-valued random vectors with $E \xi_{1}=\mathbb{O}$ and $E\left\|\xi_{1}\right\|^{2}<\infty$. If

$$
\sigma^{2}=E\left\|\xi_{1}\right\|^{2}+2 \sum_{i=2}^{\infty} \sum_{j=1}^{d} E\left(\xi_{1 j} \xi_{i j}\right)<\infty
$$

then, as $n \rightarrow \infty$

$$
n^{-\frac{1}{2}} \sum_{i=1}^{[n t]} \xi_{i} \Rightarrow W^{d}
$$

where $W^{d}$ is a d-dimensional Wiener process with covariance matrix $\Gamma=\left[\sigma_{k j}\right]$,

$$
\sigma_{k j}=E\left(\xi_{1 k} \xi_{1 j}\right)+\sum_{i=2}^{\infty}\left[E\left(\xi_{1 k} \xi_{i j}\right)+E\left(\xi_{1 j} \xi_{i k}\right)\right]
$$

From Definition 2.2 we consider the following notion:

Definition 2.4 (Burton et al. [6]). Let $\left\{\xi_{i}, i \geq 1\right\}$ be a sequence of random variables taking values in a separable Hilbert space $H .\left\{\xi_{i}, i \geq 1\right\}$ is called associated if for some orthonormal basis $\left\{e_{k}, k \geq 1\right\}$ in $H$ and for any $d \geq 1$ the $d$-dimensional sequence $\left(\left\langle\xi_{i}, e_{1}\right\rangle, \ldots,\left\langle\xi_{i}, e_{d}\right\rangle\right), i \geq 1$, is associated.

Definition 2.5 (Burton et al. [6]). Let $\left\{\xi_{i}, i \geq 1\right\}$ be a strictly stationary associated sequence $H$-valued random variables with $E \xi_{1}=0$ and $E\left\|\xi_{1}\right\|^{2}<\infty$. If

$$
\sigma^{2}=E\left\|\xi_{1}\right\|^{2}+2 \sum_{i=2}^{\infty} E\left(\left\langle\xi_{1}, \xi_{i}\right\rangle\right)<\infty
$$

then

$$
n^{-\frac{1}{2}} \sum_{i=1}^{[n t]} \xi_{i} \Rightarrow W
$$


where $W$ is a Wiener process on $H$ with covariance operator $\Gamma=\left(\sigma_{k l}\right), k, l=$ $1,2, \ldots$,

$$
\begin{aligned}
\sigma_{k l}= & E\left(\left\langle e_{k}, \xi_{1}\right\rangle\left\langle e_{l}, \xi_{1}\right\rangle\right)+\sum_{i=2}^{\infty}\left[E\left(\left\langle e_{k}, \xi_{1}\right\rangle\left\langle e_{l}, \xi_{i}\right\rangle\right)\right. \\
& \left.+E\left(\left\langle e_{l}, \xi_{1}\right\rangle\left\langle e_{k}, \xi_{i}\right\rangle\right)\right] .
\end{aligned}
$$

\section{Main results}

To prove the main theorem we need the following lemmas:

Lemma 3.1. Let $\left\{\xi_{k}, k \in \mathbb{Z}\right\}$ be a strictly stationary associated sequence of $H$ valued random variables with $E \xi_{1}=0$ and $E\left\|\xi_{1}\right\|^{2}<\infty$ and $\left\{c_{k}\right\}$ be a sequence of bounded linear operators satisfying

$$
\sum_{j=-\infty}^{\infty}\left\|c_{j}\right\|_{L(H)}<\infty .
$$

If (2.5) holds, then there is a constant $K$ such that, for every $-\infty<p<q<\infty$,

$$
E\left\|\sum_{j=p}^{q} c_{j} \xi_{j}\right\|_{H}^{2} \leq K\left(\sum_{j=p}^{q}\left\|c_{j}\right\|_{L(H)}^{2}\right) .
$$

Proof. By stationarity, (2.5), and the facts that $\left\|c_{j} \xi_{j}\right\|_{H} \leq\left\|c_{j}\right\|_{L(H)}\left\|\xi_{j}\right\|_{H}$ and $E\left(\left\langle\xi_{i}, \xi_{j}\right\rangle\right) \geq 0$ we have

$$
\begin{aligned}
E\left\|\sum_{j=p}^{q} c_{j} \xi_{j}\right\|_{H}^{2} \leq & \sum_{j=p}^{q}\left\|c_{j}\right\|_{L(H)}^{2} E\left\|\xi_{j}\right\|_{H}^{2} \\
& +2 \sum_{i=p}^{q-1} \sum_{j=i+1}^{q}\left\|c_{i}\right\|_{L(H)}\left\|c_{j}\right\|_{L(H)} E\left(\left\langle\xi_{i}, \xi_{j}\right\rangle\right) \\
\leq & \sum_{j=p}^{q}\left\|c_{j}\right\|_{L(H)}^{2} E\left\|\xi_{j}\right\|_{H}^{2}+2 \sum_{j=2}^{\infty} E\left\langle\xi_{1}, \xi_{j}\right\rangle\left(\sum_{j=p}^{q}\left\|c_{j}\right\|_{L(H)}^{2}\right) \\
\leq & K\left(\sum_{j=p}^{q}\left\|c_{j}\right\|_{L(H)}^{2}\right) .
\end{aligned}
$$

Lemma 3.2. Let $\left\{b_{k}, k \in \mathbb{Z}\right\}$ be a sequence of bounded linear operators in a Hilbert space $\left(H,\|\cdot\|_{H}\right)$ such that

$$
\sum_{k=-\infty}^{\infty}\left\|b_{k}\right\|_{L(H)}<\infty
$$


and

$$
\sum_{k=-\infty}^{\infty} b_{k}=0
$$

Then we have

$$
\frac{1}{n} \sum_{j=-\infty}^{\infty}\left\|\sum_{i=1-j}^{n-j} b_{i}\right\|_{L(H)}^{2} \rightarrow 0 \text { as } n \rightarrow \infty .
$$

Proof. Denote by $D_{n}=\sum_{|j| \geq n}\left\|b_{j}\right\|_{L(H)}$. By taking into account (3.3) we observe that

$$
\begin{aligned}
\frac{1}{n} \sum_{|j| \geq 2 n}\left\|\sum_{i=1-j}^{n-j} b_{i}\right\|_{L(H)}^{2} & \leq\left(\sum_{|j| \geq n}\left\|b_{j}\right\|_{L(H)}\right) \frac{1}{n} \sum_{j=-\infty}^{\infty}\left(\sum_{i=1-j}^{n-j}\left\|b_{i}\right\|_{L(H)}\right) \\
& =\quad D_{n} \sum_{j=-\infty}^{\infty}\left\|b_{j}\right\|_{L(H)} \rightarrow 0 \text { as } n \rightarrow \infty
\end{aligned}
$$

Now for a fixed $x$ in the interval $[-2,2]$, we define

$$
h_{n}(x)=\left\|\sum_{i=1-[n x]}^{n-[n x]} b_{i}\right\|_{L(H)}^{2} .
$$

One can easily see that, under the conditions (3.3) and (3.4), for every $x \neq 1$ we have $h_{n}(x) \rightarrow 0$, as $n \rightarrow \infty$ and $0 \leq h_{n}(x) \leq\left(\sum_{j=-\infty}^{\infty}\left\|b_{i}\right\|_{L(H)}\right)^{2}$. Hence by Lebesgue's dominated convergence theorem, we obtain

$$
\frac{1}{n} \sum_{j=-2 n}^{2 n-1}\left\|\sum_{i=1-j}^{n-j} b_{i}\right\|_{H}^{2}=\int_{0}^{2} h_{n}(x) d x \rightarrow 0 \text { as } n \rightarrow \infty .
$$

Therefore the conclusion (3.5) is a consequence of (3.6) and (3.7).

Theorem 3.3. Let $\left\{\xi_{k}, k \in \mathbb{Z}\right\}$ be a strictly stationary associated sequence of $H$-valued random variables with $E \xi_{1}=0$ and $E\left\|\xi_{1}\right\|^{2}<\infty$. Let $\left\{a_{k}, k \in \mathbb{Z}\right\}$ be a sequence of linear bounded operators such that

$$
\sum_{j=-\infty}^{\infty}\left\|a_{j}\right\|_{L(H)}<\infty
$$

If (2.5) holds, then

$$
\frac{\sum_{k=1}^{[n t]} X_{k}}{\sqrt{n}} \Rightarrow W
$$

where $X_{k}$ is defined by (1.1), $W$ is a Wiener process on $H$ with covariance operator $A \Gamma A^{*}, \Gamma$ is defined in Theorem 2.5, $A=\sum_{j=-\infty}^{\infty} a_{j}$ and $A^{*}$ denotes the adjoint operator of $A$. 
Proof. First note that by Theorem 2.5 we have

$$
\frac{A \sum_{k=1}^{[n t]} \xi_{k}}{\sqrt{n}} \rightarrow^{\mathcal{D}} W
$$

where $W$ is a Wiener process on $H$ with covariance operator $A \Gamma A^{*}$ and that from (1.1) we have

$$
\sum_{k=1}^{[n t]} X_{k}=\sum_{k=1}^{[n t]} \sum_{m=-\infty}^{\infty} a_{m} \xi_{k-m}=\sum_{j=-\infty}^{\infty}\left(\sum_{k=1}^{[n t]} a_{k-j}\right) \xi_{j} .
$$

It remains to show that

$$
n^{-\frac{1}{2}}\left\|\sum_{k=1}^{[n t]} X_{k}-A \sum_{j=1}^{[n t]} \xi_{j}\right\| \rightarrow^{p} 0
$$

by Billingsley [2], Theorem 4.1.

By partitioning the last sum in (3.11) into two sums, one with $j$ between 1 and $n$, and another containing all the other terms, we get the representation

$$
\sum_{k=1}^{[n t]} X_{k}-A \sum_{j=1}^{[n t]} \xi_{j}=\sum_{j=-\infty}^{\infty}\left(\sum_{k=1}^{[n t]} b_{k-j}\right) \xi_{j}
$$

where

$$
b_{0}=a_{0}-A \text { and } b_{i}=a_{i} \text { for }|i| \geq 1 .
$$

Now by Lemma 3.1 and Fatou Lemma, we deduce from (3.13)

$$
\begin{aligned}
& \frac{1}{n} E\left\|\sum_{k=1}^{[n t]} X_{k}-A \sum_{j=1}^{[n t]} \xi_{j}\right\|_{H}^{2} \\
\leq & \frac{1}{[n t]} E\left\|\sum_{k=1}^{[n t]} X_{k}-A \sum_{j=1}^{[n t]} \xi_{j}\right\|_{H}^{2} \\
= & \frac{1}{[n t]} E\left\|\sum_{j=-\infty}^{\infty}\left(\sum_{k=1}^{[n t]} b_{k-j}\right) \xi_{j}\right\|_{H}^{2} \\
\leq & K \frac{1}{[n t]} \sum_{j=-\infty}^{\infty}\left\|\sum_{k=1}^{[n t]} b_{k-j}\right\|_{H}^{2} \\
= & K \frac{1}{[n t]} \sum_{j=-\infty}^{\infty}\left\|\sum_{i=1-j}^{[n t]-j} b_{i}\right\|_{L(H)}^{2} .
\end{aligned}
$$

Notice that the operators $\left\{b_{i}, i \in \mathbb{Z}\right\}$ being defined by (3.14) satisfy the conditions of Lemma 3.2. Therefore from (3.15), (3.12) follows by applying Lemma 3.2 . 
Remark. Obviously, Theorem 3.3 is an extension of Theorem 1.1 to a Hilbert space.

From Theorem 3.3 we obtain the following result.

Corollary 3.4 (Kim et al. [9]). Let $\left\{\xi_{k}, k \in \mathbb{Z}\right\}$ be a strictly stationary associated sequence of $\mathbb{R}^{d}$-valued random vectors with $E \xi_{1}=\mathbb{O}$ and $E\left\|\xi_{1}\right\|^{2}<$ $\infty$ and let $\left\{B_{j}\right\}$ be a sequence of matrix such that

$$
\sum_{j=-\infty}^{\infty}\left\|B_{j}\right\|<\infty \sum_{j=-\infty}^{\infty} B_{j} \neq \mathbb{O}_{d \times d}
$$

where for any $d \times d$ matrix $B=\left(a_{i j}\right),\|B\|=\sum_{i=1}^{d} \sum_{i=1}^{d}\left|a_{i j}\right|$ and $\mathbb{O}_{d \times d}$ denotes the $d \times d$ zero matrix. Define $X_{k}$ an $\mathbb{R}^{d}$-valued linear process of the form $X_{k}=\sum_{j=-\infty}^{\infty} B_{j} \xi_{k-j}$. If (2.2) holds, then

$$
\frac{1}{\sqrt{n}} \sum_{k=1}^{[n t]} X_{k} \Rightarrow W^{d},
$$

where $W^{d}$ is a d-dimensional Wiener process with covariance matrix $T=$ $\left(\sum_{j=-\infty}^{\infty} A_{j}\right) \Gamma\left(\sum_{j=-\infty}^{\infty} A_{j}\right)^{\prime}$ and $\Gamma$ is defined in $(2.4)$.

\section{References}

[1] R. E. Barlow and F. Proschan, Statistical Theory of Reliability and Life Testing : Probability models Holt, Rinehart and Winston, New York, 1975.

[2] P. Billingsley, Convergence of Probability, John Wiley, New York, 1968.

[3] D. Bosq, Linear Processes in Function Spaces, Lectures Notes in Statistics, 149, Springer, Berlin, 2000.

[4] , Berry-Esseen inequality for linear processes in Hilbert spaces, Statist. Probab. Letters 63 (2003), 243-247.

[5] P. Brockwell and R. Davis, Time Series, Theory and Method. Springer, Berlin, 1987.

[6] R. A. R. Burton and H. Dehling, An invariance principle for weakly associated random vectors, Stochastic Processes Appl. 23 (1986), 301-306.

[7] J. T. Cox and G. Grimmett, Central limit theorems for associated random variables and the percolation model, Ann. Probab. 12 (1984), 514-528.

[8] N. A. Denisevskii and Y. A. Dorogovtsev, On the law of large numbers for a linear process in Banach space, Soviet Math. Dokl. 36 (1988), no. 1, 47-50.

[9] T. S. Kim, M. H. Ko, and S. M. Chung, A central limit theorem for the stationary multivariate linear processes generated by associated random vectors, Commun. Korean Math. Soc. 17 (2002), no. 1, 95-102.

[10] T. S. Kim and M. H. Ko, On a functional central limit theorem for stationary linear processes generated by associated processes, Bull. Korean Math. Soc. 40 (2003), no. 4, $715-722$.

[11] C. M. Newman, Normal fluctuations and the FKG inequalities, Comm. Math. Phys. 74 (1980), 119-128.

[12] C. M. Newman and A. L. Wright, An invariance principle for certain dependent sequences, Ann. Probab. 9 (1981), 671-675. 
Mi-Hwa Ko

Department of Mathematics

WONKWANG UNIVERSITY

JEONBUK 570-749, KoREA

E-mail address: songhack@wonkwang.ac.kr

TAE-Sung Kim

Department of Mathematics

WONKWANG UNIVERSITY

JEONBUK 570-749, KoreA

E-mail address: starkim@wonkwang.ac.kr 